\title{
Reversing RAFT Polymerization: Near-Quantitative Monomer Generation via a Catalyst- Free Depolymerization Approach
}

\author{
Hyun Suk Wang, ${ }^{1}$ Nghia P. Truong, ${ }^{1}$ Athina Anastasaki ${ }^{1 *}$ \\ ${ }^{1}$ Laboratory of Polymeric Materials, Department of Materials, ETH Zurich, Vladimir-Prelog-Weg 5, Zurich, \\ Switzerland
}

\begin{abstract}
The ability to reverse controlled radical polymerization and regenerate the monomer would be highly beneficial for both fundamental research and applications, yet has remained very challenging to achieve. Herein, we report a near-quantitative (up to 92\%) and catalyst-free depolymerization of various linear, bulky, crosslinked, and functional polymethacrylates made by reversible addition-fragmentation chaintransfer (RAFT) polymerization. Key to our approach is to exploit the high end-group fidelity of RAFT polymers to generate chain-end radicals via thermal homolytic cleavage of carbon-sulfur bond of the RAFT end-group at $120{ }^{\circ} \mathrm{C}$. These radicals trigger a rapid unzipping of both conventional (e.g. poly(methyl methacrylate)) and bulky polymers (e.g. poly(oligo(ethylene glycol) methyl ether methacrylate) (POEGMA)). Importantly, the depolymerization product can be utilized to either reconstruct the linear polymer or create an entirely new insoluble gel that can also be subjected to depolymerization. This work expands the potential of polymers made by CRP, pushes the boundaries of depolymerization, offers intriguing mechanistic aspects, and enables new applications.
\end{abstract}


Controlled radical polymerization (CRP), also referred to as reversible deactivation radical polymerization, has innovated the field of polymer science by providing access to well-defined polymers with tunable molecular weight, molar mass distributions, block sequence, and architecture. ${ }^{1-5}$ Perhaps the most key feature of CRP is the possibility to synthesize polymers with very high end-group fidelity which can, in turn, enable the preparation of block copolymers for use in a variety of fields including, among others, polymer self-assembly in bulk and solution. ${ }^{6-9}$ Of the CRP strategies, reversible additionfragmentation chain-transfer (RAFT) $)^{10-11}$ polymerization and atom transfer radical polymerization $(\text { ATRP })^{12}$ are arguably the most versatile and widely utilized methods. ${ }^{13}$ The former operates through a degenerative chain transfer process while the latter through a reversible termination mechanism, both of which allow efficient regulation of the equilibrium between active and dormant species and thereby achieve high livingness. ${ }^{13}$

Although such high end-group functionality has been overwhelmingly exploited for chain extensions and block copolymer synthesis in thousands of publications, it has rarely been explored for the polar opposite: reversing controlled radical polymerization and regenerating the monomer. The ability to reverse CRP and regenerate the starting materials would be highly beneficial for fundamental polymer chemistry by revealing unexplored mechanistic aspects and also significantly expand the pool of potential applications. For instance, re-obtaining the starting monomer would not only allow the ability to re-polymerize the monomer back to the initial polymer, but also provide an alternative to modify the original polymer properties or create an entirely different material (e.g. different composition, molecular weight, dispersity, architecture, etc.) tailored to a different application. This feature can also further facilitate industrial commercialization of CRP materials by offering an important innovation. In fact, IUPAC has recently acknowledged such depolymerization processes as one of the top ten emerging technologies in chemistry. ${ }^{14}$

The depolymerization of vinyl polymers is, however, not an easy task due to the highly stable carboncarbon bonds of the backbone. ${ }^{15-16}$ Current reports on reversing CRP are rather scarce and almost exclusively require the use of a metal catalyst, often yield low depolymerization conversions, and are 

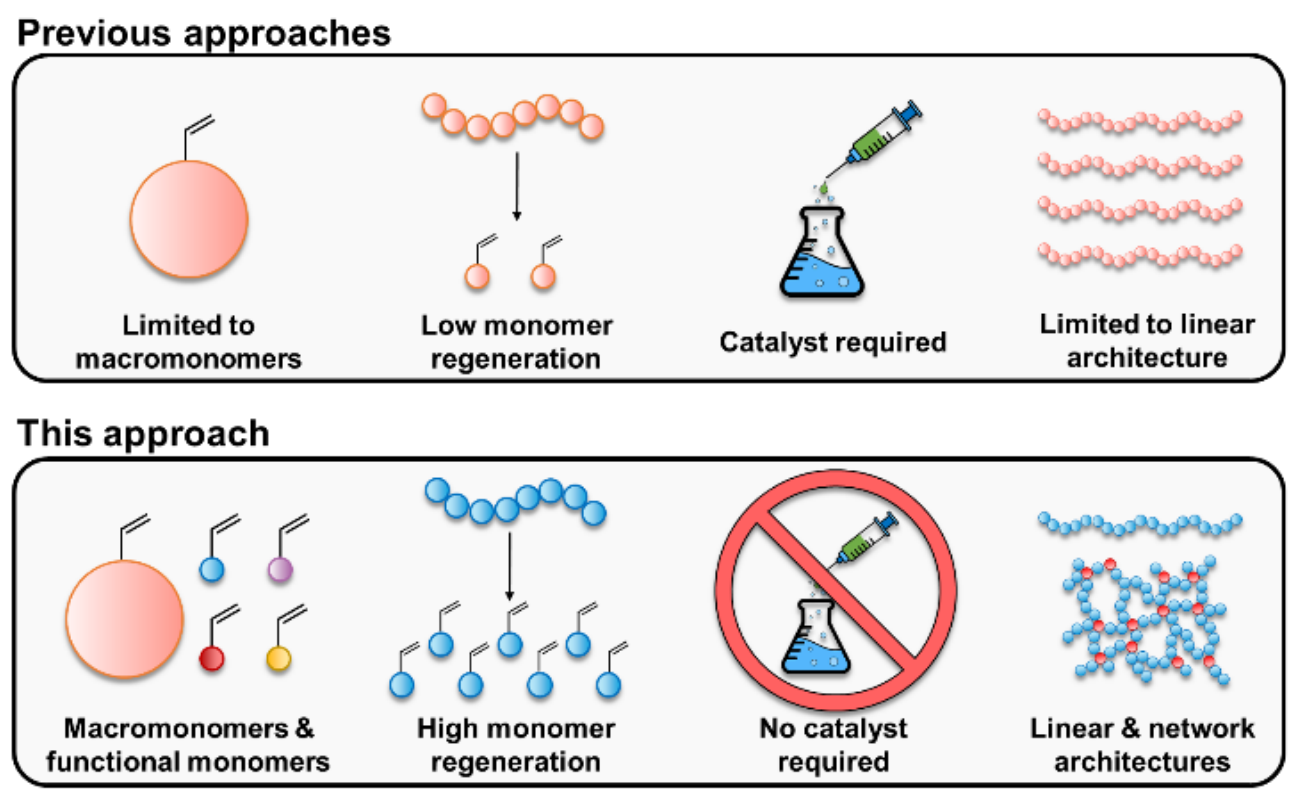

Figure 1. Illustrative comparison of our approach to previous approaches to reversing controlled radical polymerization.

limited to linear and bulky bottlebrush polymers (Figure 1). ${ }^{17-21}$ For example, Haddleton and co-workers previously described the remarkable depolymerization of water-soluble polymers in the presence of a metal catalyst and dissolved $\mathrm{CO}_{2} \cdot{ }^{17}$ However, the depolymerization mechanism was not established and depolymerization was only feasible in situ following a successful polymerization (i.e. depolymerization of isolated polymers was not demonstrated). In another interesting report by Ouchi's group, a Cl-capped PMMA could be depolymerized in the presence of a ruthenium catalyst, yielding relatively moderate depolymerization conversions (e.g. $<10 \%$ ) while side reactions were reported at higher conversions (e.g. 24\%). ${ }^{18}$ More recently, Matyjaszewski and co-workers enabled the depolymerization of a bottlebrush polymer (i.e. Cl-capped poly(poly(dimethylsiloxane) methacrylate) by utilizing a highly active copper catalyst $\left(\mathrm{CuCl}_{2} /\right.$ tris(2-pyridylmethyl)amine) at $170{ }^{\circ} \mathrm{C} .{ }^{21}$ These pioneering reports demonstrate that reversing CRP is possible and have inspired our current contribution. Herein, we report a catalyst-free and near-quantitative (up to 92\%) depolymerization methodology for both bulky and non-bulky polymethacrylates as well as insoluble gel-type materials synthesized by RAFT polymerization (Figure 1, 2a). 
Our hypothesis was that the presence of the RAFT polymer end-group would allow us to overcome the energy barrier of forming a chain-end radical and trigger depolymerization under relatively mild conditions (well below typical depolymerization temperatures of commercial polymers). ${ }^{22-23}$ In contrast, polymers made by free radical polymerization do not have such enabling end-groups and thus must overcome a significant energetic barrier to form a polymeric radical that triggers depolymerization. Indeed, the depolymerization of PMMA made by free radical polymerization is usually achieved at temperatures above $400{ }^{\circ} \mathrm{C} .{ }^{22-23}$

To test our hypothesis, a PMMA macro chain-transfer agent (macroCTA) was first synthesized via RAFT polymerization using 2-cyano-2-propyl dithiobenzoate as the chain transfer agent, yielding a well-defined polymer with narrow molar mass distribution $(\nexists=1.13)$ (Figure S1-2, Table S1). To confirm high endgroup fidelity, the purified macroCTA was chain-extended with a second monomer (benzyl methacrylate) and $10 \mathrm{~mol} \%$ azobisisobutyronitrile (AIBN) at $70^{\circ} \mathrm{C}$. A clear shift in the size exclusion chromatography

(a)

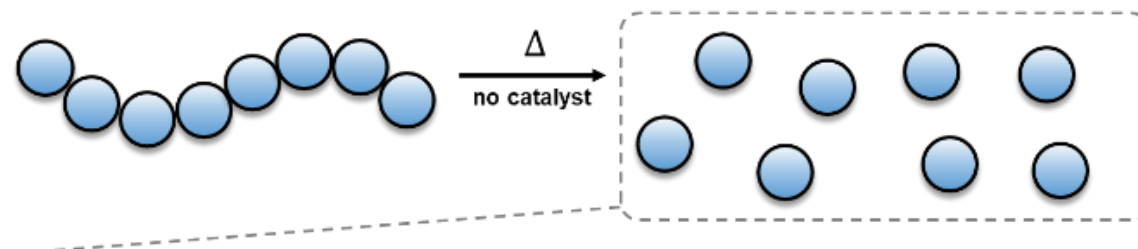

(b)

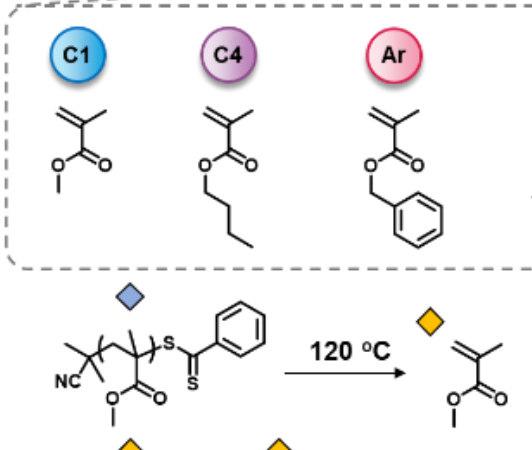

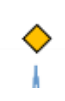

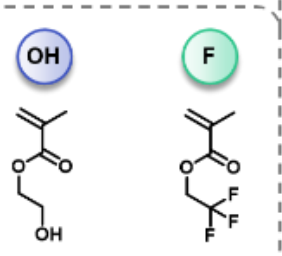

(c)

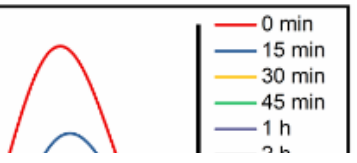

Figure 2. (a) Schematic illustration of reversing RAFT polymerization for various polymethacrylates. (b) ${ }^{1} \mathrm{H}-\mathrm{NMR}$ spectrum of the depolymerization reaction $\left(5 \mathrm{mM}, 120^{\circ} \mathrm{C}\right)$ for PMMA at various timepoints. The relative increase in the intensity of vinyl signals compared with that of the backbone confirms the regeneration of MMA. (c) Corresponding normalized SEC traces of PMMA during depolymerization. Intensities are normalized to an internal standard to portray the decrease in polymer mass during the reaction. 
(SEC) trace and low dispersity $(D=1.07)$ confirmed the high end-group fidelity of our starting material (Figure S3).

The PMMA macroCTA was then exposed to various depolymerization conditions (Figure S4) and after judicious tuning of the reaction parameters, we found that $120^{\circ} \mathrm{C}$ and $5 \mathrm{mM}$ of MMA repeat unit in dioxane (deoxygenated by nitrogen sparging) is optimal to facilitate an efficient depolymerization reaction. In particular, after $15 \mathrm{~min}$, the reaction was sampled and ${ }^{1} \mathrm{H}-\mathrm{NMR}$ showed the unambiguous reappearance of vinylidene peaks corresponding to $16 \%$ of depolymerization conversion, thus indicating that the polymerization of PMMA with dithiobenzoate end groups was indeed reversed (Figure 2b). Careful kinetic evaluation showed that the intensity of the vinylidene peaks continued increasing with time, reaching a maximum depolymerization conversion of $86 \%$ after 8 h (Figure 2 b). SEC also confirmed the successful depolymerization of PMMA, as evident by the significant decrease of the intensity of the polymer peak (normalized to an internal control polymer) (Figure 2c, S5). Interestingly, the molar mass distribution did not shift significantly throughout the depolymerization, thus suggesting rapid and complete unzipping of activated polymer chains to regenerate the monomer (Table S2). To the best of our knowledge, this is the highest reported depolymerization conversion of non-bulky monomers via reversing CRP. Notably, to recover both the regenerated monomer and solvent, a high-boiling solvent such as dimethylsulfoxide can be alternatively employed, enabling the fractional distillation of the regenerated MMA and also the dimethylsulfoxide, both at 99\% purity (Figure S6-7). Importantly, our depolymerization approach operates in the absence of any catalyst and at significantly lower temperatures compared to polymers synthesized by free radical polymerization. This data further validates our hypothesis and suggest that the RAFT end-group is responsible for the efficient depolymerization observed.

To shine a light on the depolymerization mechanism, the PMMA RAFT end-group was removed using a previously established protocol ${ }^{24}$ in which an excess of AIBN was employed at $70{ }^{\circ} \mathrm{C}$ (Figure S8). The dithiobenzoate-free PMMA was then exposed to identical depolymerization conditions. As expected, 
negligible $(<3 \%)$ depolymerization could be observed within the same $8 \mathrm{~h}$ timeframe, thus strongly supporting our initial hypothesis that the RAFT end-group is essential to trigger an efficient depolymerization. To further investigate the mechanistic pathway, the radical scavenger 2,2,6,6tetramethylpiperidin-1-yl)oxyl (TEMPO) was added to the solution (PMMA dissolved in dioxane) prior to depolymerization. In the presence of the radical scavenger, very minimal depolymerization was observed $(<5 \%)$ thereby indicating that depolymerization occurred via a radical pathway (Figure S9). A key remaining question was to identify the origin of the chain-end radicals as the reaction was performed in the absence of any known radical-generating species such as AIBN. It has been reported that a chain-end radical can be formed via a photo-induced homolytic cleavage of the RAFT end-group from the polymer chain end (i.e. cleaving of the C-S bond). ${ }^{25-27}$ To explore the possibility of a thermally-induced homolytic cleavage of the RAFT end-group, additional experiments were conducted. If this was a viable theory, in the presence of monomer, the macroCTA PMMA would be expected to undergo chain extension at $120^{\circ} \mathrm{C}$ in the absence of a free radical initiator. To exclude the possibility of radical generation through the solvent, the chain extension reaction was performed in bulk. Upon adding a second monomer (benzyl methacrylate), a clear shift to a higher molar mass distribution with low dispersity $(\nexists=1.18)$ was evident by SEC (Figure S10). Simply heating up the monomer at $120{ }^{\circ} \mathrm{C}$ did not yield to any self-initiated autopolymerization (Figure S11), indicating that no radicals were generated thermally by benzyl methacrylate. Altogether, these results suggest that the chain-end radical was formed via homolytic C-S cleavage at $120^{\circ} \mathrm{C}$ and subsequently triggered the rapid and near-quantitative depolymerization of PMMA.

The scope of our depolymerization approach was then extended to a number of other methacrylic polymers synthesized by RAFT polymerization including poly(oligo(ethylene glycol) methyl ether methacrylate) (POEGMA), poly(butyl methacrylate) (PBuMA) and poly(benzyl methacrylate) (PBzMA) (Figure S12-17). All polymers were successfully depolymerized, achieving 87\%, 92\% and 92\% conversions, respectively, representing the highest conversion reported to date for any CRP depolymerization strategy (Figure 3). A series of more functional and challenging polymers were also 

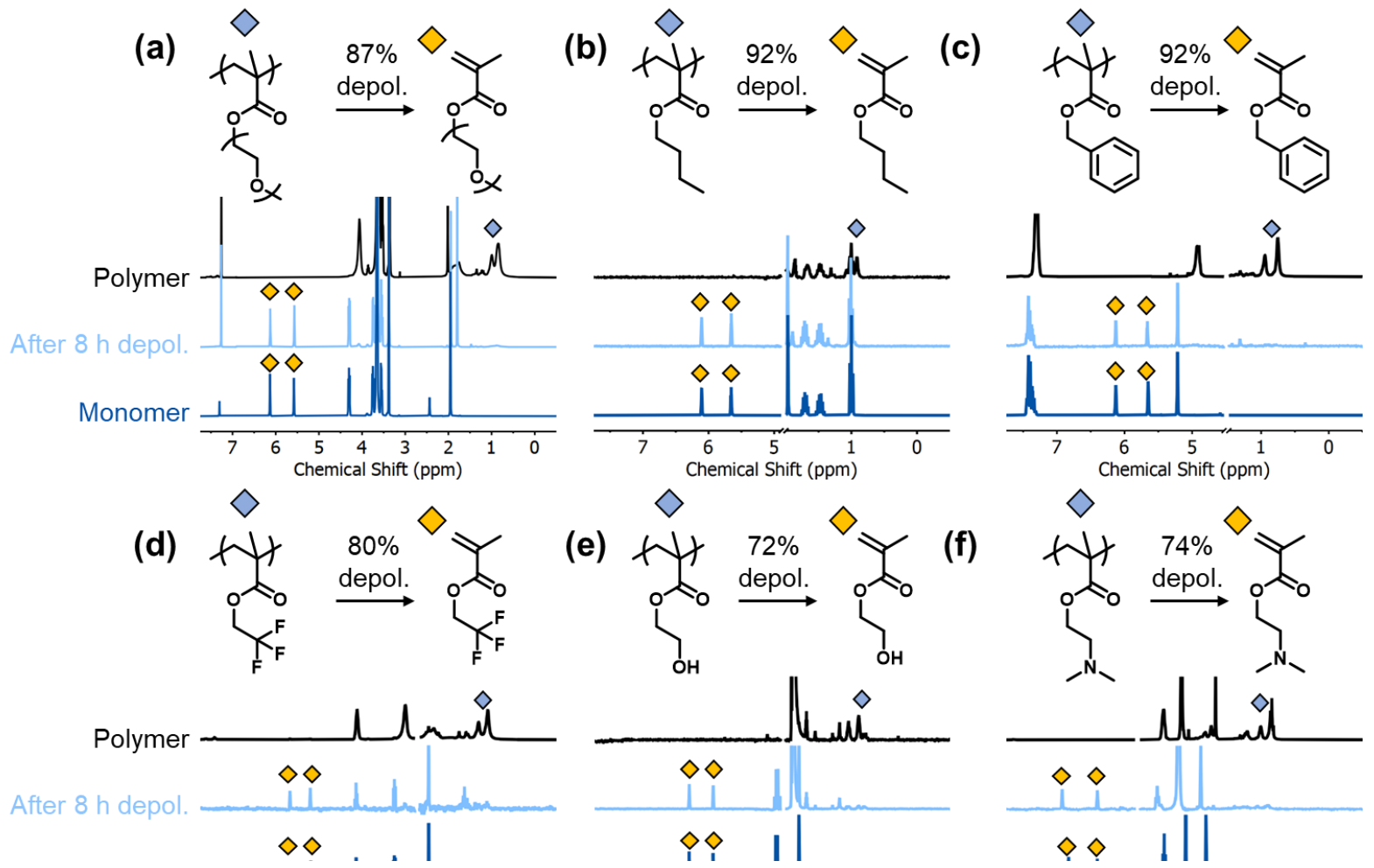

Figure 3. ${ }^{1} \mathrm{H}-\mathrm{NMR}$ spectrum of the polymer (top spectrum), reaction mixture after depolymerization (middle spectrum), and the original monomer (bottom spectrum) for (a) POEGMA, (b) PBuMA, (c) PBzMA, (d) PTFEMA, (e) PHEMA, and (f) PDMAEMA. Reactions were run in $5 \mathrm{mM}$ concentration and $120^{\circ} \mathrm{C}$ for $8 \mathrm{~h}$. Dioxane was used as the solvent in all cases except for PHEMA for which dimethylsulfoxide was used.

successfully subjected under our optimized depolymerization conditions such as the fluorine-containing poly(trifluoroethyl methacrylate) (PTFEMA), the pH-responsive poly(2-(dimethylamino)ethyl methacrylate) (PDMAEMA) and the hydroxyl-functionalized poly(hydroxyethyl methacrylate) (PHEMA) (Figure S18-23) yielding high percentages of regenerated monomer (Figure 3). To demonstrate the potential of our methodology to reuse the resulting depolymerization mixture, we chose POEGMA as a model water-soluble polymer as this is also a promising material for making hydrogels which we will further explore later in the manuscript. POEGMA was first depolymerized to $87 \%$ conversion (Figure 4a, b) and the residual polymer was removed via precipitation in diethyl ether/hexane. The supernatant (i.e. depolymerization products excluding the precipitated polymer) was then concentrated and polymerized at $70{ }^{\circ} \mathrm{C}$ by adding $10 \mathrm{~mol} \%$ AIBN. Polymerization proceeded in a controlled manner, with the molar mass distributions gradually shifting to higher molecular weights (linear evolution of $M_{\mathrm{n}}$ with monomer 
conversion) while very low $\oslash$ values $(1.11-1.15)$ were maintained throughout the re-polymerization (Figure 4c, d). This data not only suggest that the re-polymerization of retrieved mixtures is well-controlled but also that the recycling of both the monomer and CTA is possible.

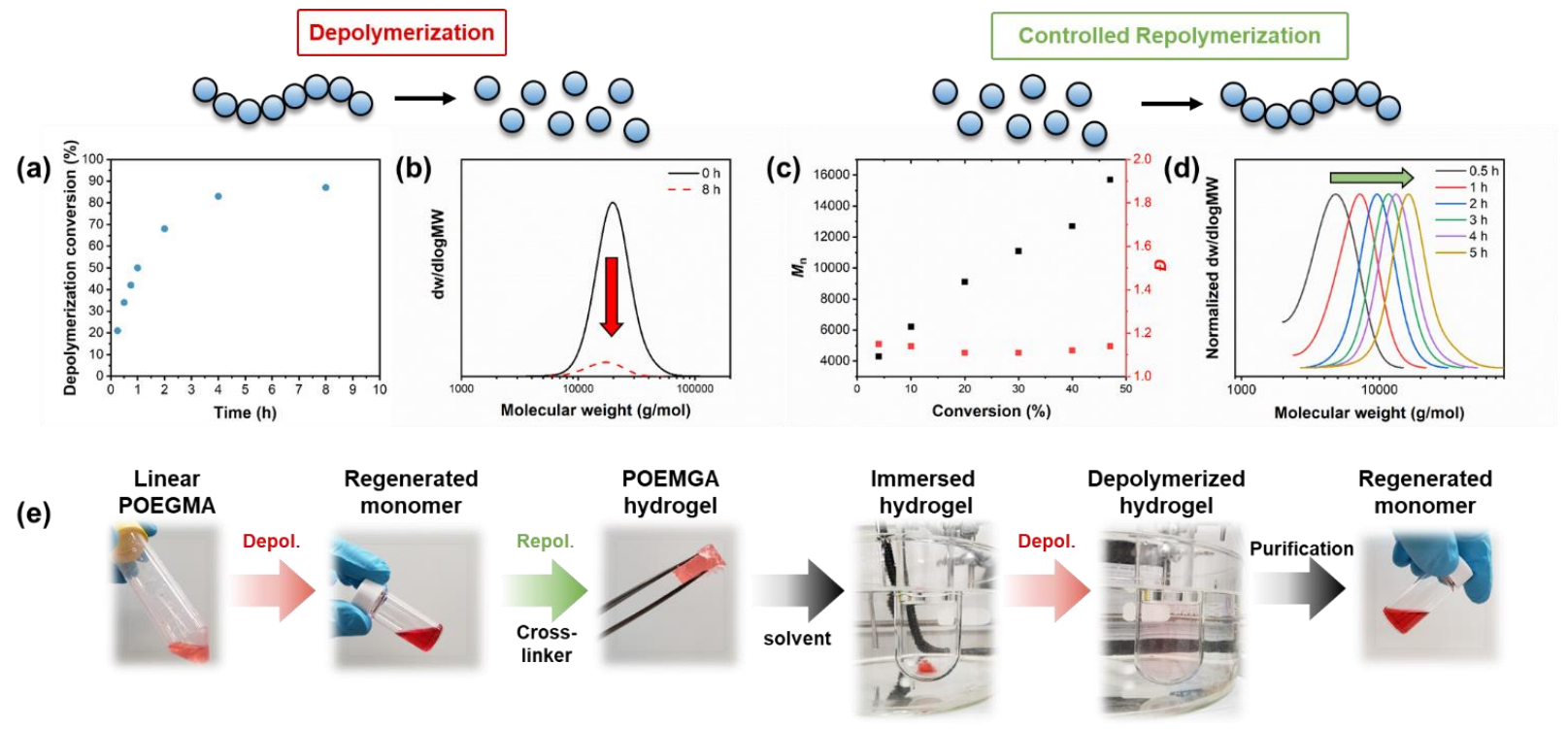

Figure 4. (a) Plot of the monomer conversion during the depolymerization of POEGMA and (b) the normalized SEC trace after $8 \mathrm{~h}$. (c) Evolution of $M_{\mathrm{n}}$ and $D$ with conversion during the controlled repolymerization of regenerated OEGMA. (d) SEC traces during controlled repolymerization. (e) Depolymerization and repolymerization steps for synthesizing an entirely new insoluble hydrogel from linear POEGMA, then subsequently depolymerizing the hydrogel to recover the monomers.

Last but not least, to probe the potential of this approach for materials application, we performed a doubledepolymerization procedure with POEGMA (Figure 4e) with the aim to not only regenerate the monomer twice but also to produce a different material in the process. In the first step, POEGMA was depolymerized to the monomer (87\%) and the residual polymer was removed via precipitation in diethyl ether/hexane. The supernatant was concentrated and subsequently polymerized into a hydrogel by adding oligo(ethylene glycol) dimethacrylate (OEGDMA) as a crosslinking comonomer ([OEGMA]:[OEGDMA] = 5:1) (see Supporting Information for details). The hydrogel was soaked in acetone for one day to remove any residual monomer and dried. In the second step, the hydrogel was immersed in dioxane $(0.35 \mathrm{~g} / 40 \mathrm{ml})$ and the solution was bubbled with $\mathrm{N}_{2}$ and heated to $120^{\circ} \mathrm{C} .4 \mathrm{~h}$ later, the mixture became a fully homogeneous pink solution, visibly evidencing successful depolymerization even from the gel state. ${ }^{1} \mathrm{H}-\mathrm{NMR}$ analysis of 
the homogenous solution after $12 \mathrm{~h}$ showed a depolymerization conversion of $71 \%$, demonstrating the first reversal of CRP for an insoluble gel. The ability to depolymerize insoluble gel-type materials is particularly promising as it indicates that dissolution of the target material is not a necessity, making this approach applicable to a wider range of materials. To confirm that the dissolution was a consequence of depolymerization and not improved solubility (i.e. that the hydrogel was indeed a fully insoluble network and not merely a high molecular weight branched polymer with limited solubility) we attempted to depolymerize a CTA-cleaved version of the gel (See Supporting Information, Figure S24). After 12 h, 83 $\mathrm{wt} \%$ of the gel remained intact (Figure S25), confirming both the networked structure of the gel and the depolymerization-induced dissolution.

In summary, we report a catalyst-free depolymerization strategy that can be applied to a wide range of linear, bulky, crosslinked, and functional polymethacrylic materials synthesized by RAFT yielding extremely high depolymerization conversions (up to $92 \%$ ). Upon heating the polymethacrylates solutions

at $120 \mathrm{C}$, the $\mathrm{C}-\mathrm{S}$ bonds are homolytically cleaved producing chain-end radicals that trigger a rapid depolymerization. By exploiting the high end-group fidelity of RAFT polymers, our method requires a much lower depolymerization temperature than that needed for polymers made by FRP (e.g. PMMA). Furthermore, the potential to reuse the recovered monomer and turn it to either a similar linear polymer or an entirely new material was also demonstrated.

\section{REFERENCES}

1. Parkatzidis, K.; Wang, H. S.; Truong, N. P.; Anastasaki, A., Recent developments and future challenges in controlled radical polymerization: A 2020 update. Chem 2020, 6 (7), 1575-1588.

2. Gentekos, D. T.; Sifri, R. J.; Fors, B. P., Controlling polymer properties through the shape of the molecular-weight distribution. Nat. Rev. Mater. 2019, 1-14.

3. Yin, R.; Wang, Z.; Bockstaller, M. R.; Matyjaszewski, K., Tuning dispersity of linear polymers and polymeric brushes grown from nanoparticles by atom transfer radical polymerization. Polym. Chem. 2021, Advance Article. 
4. Gody, G.; Maschmeyer, T.; Zetterlund, P. B.; Perrier, S., Rapid and quantitative one-pot synthesis of sequence-controlled polymers by radical polymerization. Nat. Commun. 2013, 4 (1), 2505.

5. De Neve, J.; Haven, J. J.; Maes, L.; Junkers, T., Sequence-definition from controlled polymerization: the next generation of materials. Polym. Chem. 2018, 9 (38), 4692-4705.

6. Elsabahy, M.; Wooley, K. L., Design of polymeric nanoparticles for biomedical delivery applications. Chem. Soc. Rev. 2012, 41 (7), 2545-2561.

7. Bates, C. M.; Maher, M. J.; Janes, D. W.; Ellison, C. J.; Willson, C. G., Block Copolymer Lithography. Macromolecules 2014, 47 (1), 2-12.

8. Figg, C. A.; Simula, A.; Gebre, K. A.; Tucker, B. S.; Haddleton, D. M.; Sumerlin, B. S., Polymerizationinduced thermal self-assembly (PITSA). Chem. Sci. 2015, 6 (2), 1230-1236.

9. Zhang, J.; Farias-Mancilla, B.; Kulai, I.; Hoeppener, S.; Lonetti, B.; Prévost, S.; Ulbrich, J.; Destarac, M.; Colombani, O.; Schubert, U. S.; Guerrero-Sanchez, C.; Harrisson, S., Effect of Hydrophilic Monomer Distribution on Self-Assembly of a pH-Responsive Copolymer: Spheres, Worms and Vesicles from a Single Copolymer Composition. Angew. Chem. Int. Ed. 2021, 60 (9), 4925-4930.

10. Moad, G.; Rizzardo, E.; Thang, S. H., Living Radical Polymerization by the RAFT Process. Aust. J. Chem. 2005, $58(6), 379-410$.

11. Barner-Kowollik, C., Handbook of RAFT polymerization. Wiley-VCH Verlag GmbH \& Co. KGaA: Weinheim, Germany, 2008.

12. Matyjaszewski, K., Atom Transfer Radical Polymerization (ATRP): Current Status and Future Perspectives. Macromolecules 2012, 45 (10), 4015-4039.

13. Truong, N. P.; Jones, G. R.; Bradford, K. G. E.; Konkolewicz, D.; Anastasaki, A., A comparison of RAFT and ATRP methods for controlled radical polymerization. Nat. Rev. Chem. 2021.

14. IUPAC Top Ten Emerging Technologies in Chemistry. https://iupac.org/what-we-do/top-ten/.

15. Delplace, V.; Nicolas, J., Degradable vinyl polymers for biomedical applications. Nat. Chem. 2015, 7 (10), 771-784. 
16. Coates, G. W.; Getzler, Y. D. Y. L., Chemical recycling to monomer for an ideal, circular polymer economy. Nat. Rev. Mater. 2020, 5 (7), 501-516.

17. Lloyd, D. J.; Nikolaou, V.; Collins, J.; Waldron, C.; Anastasaki, A.; Bassett, S. P.; Howdle, S. M.; Blanazs, A.; Wilson, P.; Kempe, K.; Haddleton, D. M., Controlled aqueous polymerization of acrylamides and acrylates and "in situ" depolymerization in the presence of dissolved CO2. Chem. Commun. 2016, 52 (39), 6533-6536.

18. Sano, Y.; Konishi, T.; Sawamoto, M.; Ouchi, M., Controlled radical depolymerization of chlorinecapped PMMA via reversible activation of the terminal group by ruthenium catalyst. Eur. Polym. J. 2019, 120, 109181.

19. Flanders, M. J.; Gramlich, W. M., Reversible-addition fragmentation chain transfer (RAFT) mediated depolymerization of brush polymers. Polym. Chem. 2018, 9 (17), 2328-2335.

20. Li, L.; Shu, X.; Zhu, J., Low temperature depolymerization from a copper-based aqueous vinyl polymerization system. Polymer 2012, 53 (22), 5010-5015.

21. Martinez, M. R.; Dadashi-Silab, S.; Lorandi, F.; Zhao, Y.; Matyjaszewski, K., Depolymerization of P(PDMS11MA) Bottlebrushes via Atom Transfer Radical Polymerization with Activator Regeneration. Macromolecules 2021, 54 (12), 5526-5538.

22. Godiya, C. B.; Gabrielli, S.; Materazzi, S.; Pianesi, M. S.; Stefanini, N.; Marcantoni, E., Depolymerization of waste poly(methyl methacrylate) scraps and purification of depolymerized products. J. Environ. Manage. 2019, 231, 1012-1020.

23. Kaminsky, W.; Franck, J., Monomer recovery by pyrolysis of poly(methyl methacrylate) (PMMA). $J$. Anal. Appl. Pyrolysis 1991, 19, 311-318.

24. Willcock, H.; O'Reilly, R. K., End group removal and modification of RAFT polymers. Polym. Chem. 2010, 1 (2), 149-157.

25. Carmean, R. N.; Sims, M. B.; Figg, C. A.; Hurst, P. J.; Patterson, J. P.; Sumerlin, B. S., Ultrahigh Molecular Weight Hydrophobic Acrylic and Styrenic Polymers through Organic-Phase PhotoiniferterMediated Polymerization. ACS Macro Lett. 2020, 9 (4), 613-618. 
26. Carmean, R. N.; Becker, T. E.; Sims, M. B.; Sumerlin, B. S., Ultra-High Molecular Weights via Aqueous Reversible-Deactivation Radical Polymerization. Chem 2017, 2 (1), 93-101.

27. Figg, C. A.; Hickman, J. D.; Scheutz, G. M.; Shanmugam, S.; Carmean, R. N.; Tucker, B. S.; Boyer, C.; Sumerlin, B. S., Color-Coding Visible Light Polymerizations To Elucidate the Activation of Trithiocarbonates Using Eosin Y. Macromolecules 2018, 51 (4), 1370-1376. 